\title{
Primary syphilis of the eyelid: case report
}

\author{
W JEYAKUMAR, A CHITHRA, A SHANMUGASUNDARARAJ \\ From the Department of Sexually Transmitted Diseases, Government Rajaji Hospital, Madurai, Tamilnadu, \\ India
}

SUMMARY Primary syphilis of the palpebral conjunctiva of the lower eyelid is reported because it is rare, which makes its diagnosis difficult but important.

Reports of primary, secondary, or gummatous lesions of the conjunctiva have been published only sporadically, though syphilis is considered to be one of the commonest sexually transmitted diseases (STD) in developing countries.

Transmission may be by infected fingers, handkerchiefs, towels, gloves, sneeze or cough droplets, or a tongue used to remove a foreign body from the eye. The other possible differential diagnoses are leptospirosis, lymphogranuloma venereum, and tuberculosis. Chancre of the eyelid is rarely encountered in STD departments, as patients with this lesion may be referred to ophthalmic departments with suspected chalazion. ${ }^{\prime}$

\section{Case report}

An unmarried man aged 22 was referred to the department of sexually transmitted diseases (STD), from the ophthalmology department of this hospital in January 1988 because he had an ulcer of 15 days' duration in the left eye (fig) and positive results to serological tests for syphilis. History showed that he was promiscuous but had had no previous STDs. On examination an elliptical, painless, indurated ulcer, measuring about $0.8 \times 0.5 \mathrm{~cm}$, was seen near the medial canthus of the lower palpebral conjunctiva of the left eye. His eyelashes, upper eyelid, cornea, bulbar conjunctiva, the other eye, and the rest of his face showed no abnormality. His left preauricular and submandibular nodes were enlarged, discrete, nontender, and firm. He had no genital or anal lesions.

Scrape material from the ulcer showed Treponema pallidum on dark field microscopy. The Venereal Disease Research Laboratory (VDRL) test gave a

Address for reprints: Dr W Jeyakumar, Department of Sexually Transmitted Diseases, Government Rajaji Hospital, Madurai 625020, Tamilnadu, India

Accepted for publication 2 March 1989 positive result at a titre of $1 / 16$, and the $T$ pallidum haemagglutination assay (TPHA) also gave a positive result. Gram's stain of the scrape material gave a negative result for Haemophilus ducreyi bacilli. Smear and culture studies for Neisseria gonorrhoeae, Trichomonas vaginalis, and Candida albicans gave negative results. His total blood count was $8.2 \times 10^{9} / 1$, and the differential count was $70 \%$ polymorphs, $28 \%$ lymphocytes, and $2 \%$ eosinophils. The erythrocyte sedimentation rate was $4 \mathrm{~mm}$ in the first hour. A Mantoux test gave a negative result. Analysis of the cerebrospinal fluid and radiography of the chest and long bones showed no abnormalities. Histopathological examination of the ulcer showed perivascular infiltration with lymphocytes and plasma cells and a breach in the epithelium.

The patient was treated with a single deep intramuscular injection of benzathine penicillin $2.4 \mathrm{MIU}$, and the lesion had healed completely within six days. Routine clinical and serological follow up studies undertaken during the following 10 months showed a

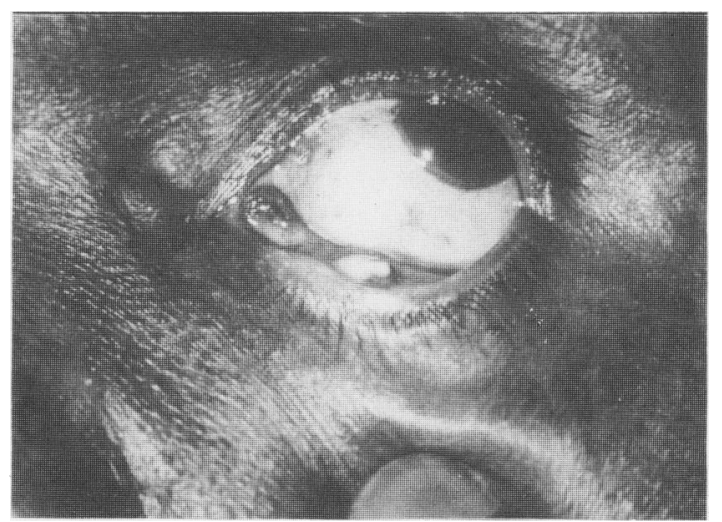

Figure Primary syphilitic chancre of lower left palpebral conjunctiva. 
complete absence of symptoms and signs and declining titres.

His sexual contacts could not be traced for epidemiological treatment.

\section{Discussion}

Chancre of the eyelid deserves a mention because it is a rare manifestation of syphilitic infection. The most common site of a primary chancre in the eye is the lower lid, ${ }^{2}$ and in our patient, the lesion was present in the lower palpebral conjunctiva. The diagnosis of syphilis was confirmed by the typical clinical picture, $T$ pallidum being shown by dark field microscopy, positive results to non-specific and specific serological tests for syphilis, histology, and the prompt response to antisyphilitic treatment. The other differential diagnoses were ruled out by appropriate investigations.

The exact aetiology of this patient's syphilis could not be ascertained. Patients with syphilis at unusual sites should be diagnosed and treated to protect themselves and others.

We thank the Dean of the Government Rajaji Hospital, Madurai, for permitting us to report on this patient, and the pathology department and the photographer, Mr Murugan, for their help.

\section{References}

1 Duke-Elder S. Parson's diseases of the eye. 15th ed. London: Churchill Livingstone, 1970:149,172,173.

2 Duke-Elder S. System of ophthalmology. Diseases of the outer eye, Vol 8. London: Henry Kimpton, 1965:238. 